\title{
Compressional sound velocity, equation of state, and constitutive response of shock-compressed magnesium oxide
}

\author{
Thomas S. Duffy ${ }^{1}$ and Thomas J. Ahrens \\ Seismological Laboratory, California Institute of Technology, Pasadena
}

\begin{abstract}
Wave profile and equation of state (EOS) data are reported for lowporosity polycrystalline magnesium oxide under shock compression. The Hugoniot equation of state between 14 and $133 \mathrm{GPa}$ is $U_{S}=6.87(10)+1.24(4) u_{p}$, where the numbers in parentheses are one standard deviation uncertainties in the last $\operatorname{digit}(\mathrm{s})$. Reverse-impact wave profiles constrain the compressional sound velocity, $V_{P}$, at $10-27 \mathrm{GPa}$ to $\pm 2 \%$. Measured $V_{P}$ values are consistent with ultrasonic data extrapolated from $3 \mathrm{GPa}$. By combining the Hugoniot results with ultrasonic data, the adiabatic bulk modulus and its first and second pressure derivatives at constant entropy are $162.5(2) \mathrm{GPa}, 4.09(9)$, and $-0.019(4) \mathrm{GPa}^{-1}$. The shear modulus and its first and second pressure derivatives are 130.8(2) $\mathrm{GPa}, 2.5(1),-0.026(45) \mathrm{GPa}^{-1}$. Polycrystalline $\mathrm{MgO}$ has a compressive yield strength of $1-1.5 \mathrm{GPa}$ at the elastic limit which increases to $2.7(8) \mathrm{GPa}$ along the Hugoniot and is similar at unloading. Wave profiles for $\mathrm{MgO}$ at 10-39 $\mathrm{GPa}$ are described using a modified elastic-plastic model. There are significant differences in the dynamic response of single-crystal and polycrystalline $\mathrm{MgO}$.
\end{abstract}

\section{Introduction}

The most direct approach for understanding the composition and structure of the Earth's deep interior is through measurement of elastic wave velocities in minerals and metals at high pressures and temperatures. Laterally averaged seismic wave velocities are known to better than 1\% throughout the Earth [Lay et al., 1990]. Small lateral deviations of wave velocities have also now been extensively documented [Romanowicz, 1991]. Under static pressure, there are few measurements of elastic wave velocities above $1 \mathrm{GPa}$ in minerals of planetary interest.

Sound velocities in shock-compressed materials were first measured by Al'tshuler et al. [1960]. Subsequent work of particular geophysical significance included the study of Fe by Brown and McQueen [1986]. Sound velocities measured under shock compression have been used to place experimental constraints on the temperature coefficient of compressional velocity at $\sim 100 \mathrm{GPa}$ [Duffy and Ahrens, 1992, 1994a]. This is an important quantity for interpretation of seismic tomography. In this study, the effect of pressure on compressional

\footnotetext{
${ }^{1}$ Now at Geophysical Laboratory, Carnegie Institution of Washington, D.C.

Copyright 1995 by the American Geophysical Union.
}

Paper number 94JB02065.

0148-0227/95/94JB-02065\$05.00 wave velocity is studied in shock-compressed $\mathrm{MgO}$ to $27 \mathrm{GPa}$. The results provide a test of finite strain extrapolation and place constraints on higher-order elastic constants. We report direct measurements of the compressional wave speed in a potentially significant lower mantle material at lower mantle pressures.

$\mathrm{MgO}$ has been the subject of extensive theoretical and experimental study as a result of its wide pressure and temperature stability range and simple structure. While single-crystal $\mathrm{MgO}$ has been studied previously under shock compression, we report the first equation of state (EOS) and wave profile measurements on low-porosity polycrystalline MgO. Significant differences in the shock response of polycrystals and single crystals have been identified in several materials [Mashimo, 1993]. There has also been much recent interest in the dynamic properties of ceramics whose response to dynamic compression appears to be quite variable but is largely unknown [Rosenberg, 1992; Mashimo, 1993]. An abbreviated report of this work is given by Duffy and Ahrens [1994b].

\section{Experimental Method}

\section{Samples}

Polycrystalline $\mathrm{MgO}$ samples were obtained (Cercom Incorporated, Vista, California) as 1.25-inch-diameter hot-pressed disks. The manufacturer's specifications indicated that the density was within $1 \%$ of crystal density and chemical purity was greater than $99.5 \%$. The latter was confirmed by electron microprobe analysis 
which revealed a small amount of $\mathrm{CaO}(\sim 0.3 \%)$ and no other impurities at detectable levels. Bulk densities were measured by weighing the $\sim 10 \mathrm{~g}$ samples with a microbalance sensitive to $\pm 10^{-4} \mathrm{~g}$. The average crystal density was found by Archimedean immersion to be $3.571(4) \mathrm{g} / \mathrm{cm}^{3}$, and the average bulk density was $3.562(6) \mathrm{g} / \mathrm{cm}^{3}$, which are within $0.4 \%$ and $0.6 \%$, respectively, of the $X$ ray density of $3.584 \mathrm{~g} / \mathrm{cm}^{3}$. The numbers in parentheses are one standard deviation uncertainties in the last digit. Optical examination of thin sections revealed a structure of colorless, roughly equant grains with approximate dimensions of $5 \mu \mathrm{m}$. Sample flatness variations were less than $0.01 \mathrm{~mm}$.

Ultrasonic sound velocity measurements were performed along the cylindrical axis (in the direction of shock wave propagation) by S. M. Rigden at Australian National University. Measurements between 30 and $70 \mathrm{MHz}$ revealed little dispersion in velocity $( \pm 0.005 \mathrm{~km} / \mathrm{s})$ and a compressional wave velocity of $9.81(2) \mathrm{km} / \mathrm{s}$. This is $1.1 \%$ greater than the VoigtReuss-Hill average for a single crystal of this material [Jackson and Niesler, 1982]. This difference exceeds the Voigt and Reuss bounds on the sound velocity and probably results from internal strains, small amounts of impurities, or preferred orientation. These effects all occur in polycrystalline $\mathrm{MgO}$ samples [Tagai et al., 1967; Schreiber and Anderson, 1968; Spetzler and Anderson, 1971].

\section{Equation of State Experiments}

Equation of state experiments were conducted using both a propellant and a light gas gun. The $40-\mathrm{mm}$ bore propellant gun could launch $\sim 100-\mathrm{g}$ projectiles to velocities up to $2.5 \mathrm{~km} / \mathrm{s}$, while the two-stage light gas gun can accelerate $\sim 20$-g projectiles to $6.5 \mathrm{~km} / \mathrm{s}$. Projectile velocity was measured by recording $X$ ray shadowgraphs immediately prior to impact. The MgO targets were mounted on tantalum or aluminum 2024 driver plates and placed in an evacuated $(\sim 100-\mathrm{mm} \mathrm{Hg})$ sample chamber. Two pairs of flat mirrors were mounted on the rear surface of the target and driver. An inclined wedge was mounted in the center of the target. Light from a 10-kV Xenon flash lamp was directed onto the rear of the target, and reflected light was returned to a continuous writing streak camera. Shock wave velocities were measured by recording the reduction in reflected light intensity due to the destruction of the mirrors by the shock front and by the change in extinction angle of the inclined wedge. Further details are given by Ahrens [1987].

\section{Wave Profile Experiments}

A series of wave profile measurements was undertaken using shock wave velocimetry. These measurements yield a continuous record of particle velocity at a sample interface during both the loading and unloading cycles of the experiment. The method for recording particle velocity histories was the velocity interferometer system for any reflector (VISAR) [Barker and Hollenbach, 1972]. In this technique, $200-300 \mathrm{~mW}$ of light from an
$\mathrm{Ar}^{+}$ion laser is focused onto a diffusely reflecting surface. Target motion induces a Doppler shift in the reflected laser light which generates interference fringes in a wide-angle Michelson interferometer. The fringes are recorded using photomultiplier tubes and digital oscilloscopes. The relationship between surface velocity and number of fringes is [Barker and Hollenbach, 1972]

$$
u(t-\tau / 2)=k F(t),
$$

where $u$ is the particle velocity, $t$ is the time, $\tau$ is the lag time ( $\sim 1 \mathrm{~ns})$ of the interferometer, $k$ is the velocityper-fringe constant, and $F(t)$ is the number of fringes recorded up to time $t$. The fringe constant $k$ can range from $\sim 100 \mathrm{~m} / \mathrm{s} /$ fringe to over $1 \mathrm{~km} / \mathrm{s} /$ fringe and is controlled by inserting fused silica etalons in one arm of the interferometer. The VISAR we constructed is similar to that described by Barker and Hollenbach [1972]. We also incorporated the push-pull modification and data reduction algorithm of Hemsing [1979] and the polarization-randomization scheme of Asay and Barker [1974].

Two types of impact geometries, forward and reverse, were used. The reverse-impact experimental setup is illustrated in Figure 1a. In this arrangement, the sample is inserted in the projectile and used to impact a thin aluminum buffer with an LiF window epoxied to it. The VISAR monitors the interface between the window and buffer. An array of electrical shorting pins is used as part of a capacitor-discharge circuit to trigger recording instrumentation and to measure impact planarity. The sample is backed by low-density foam which serves to introduce an unloading wave into the specimen.

A Lagrangian wave propagation diagram for the reverse-impact experiments is shown in Figure $1 \mathrm{~b}$. At impact $(t=0)$, an elastic precursor and a shock wave propagate through the sample, and a shock travels through the buffer. It is assumed here that the impact stress is sufficient to overdrive the precursor in aluminum but not sufficient to overdrive the precursor in $\mathrm{MgO}$. At time $t_{1}$, the shock reaches the buffer-window interface and is recorded by the VISAR. The slight impedance contrast between $\mathrm{LiF}$ and aluminum causes a weak rarefaction to propagate through the aluminum which returns to the reflecting surface at $t_{2}$. The elastic precursor traveling through the sample reaches the sample-foam interface, reflects from it as a rarefaction wave, and interacts with the oncoming shock. The shock wave also reflects from the foam interface and produces a rarefaction which fur-

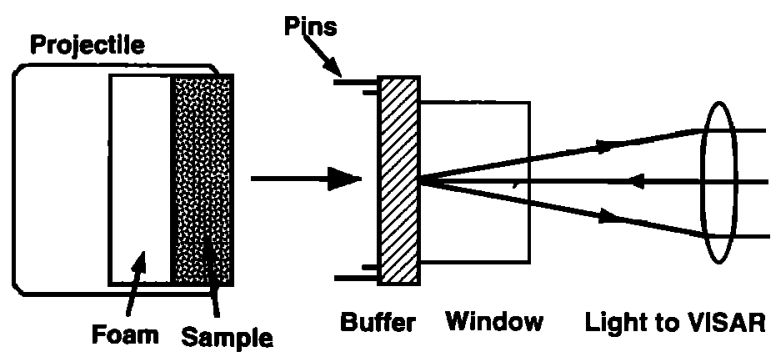

Figure 1a. Reverse-impact experimental geometry. 


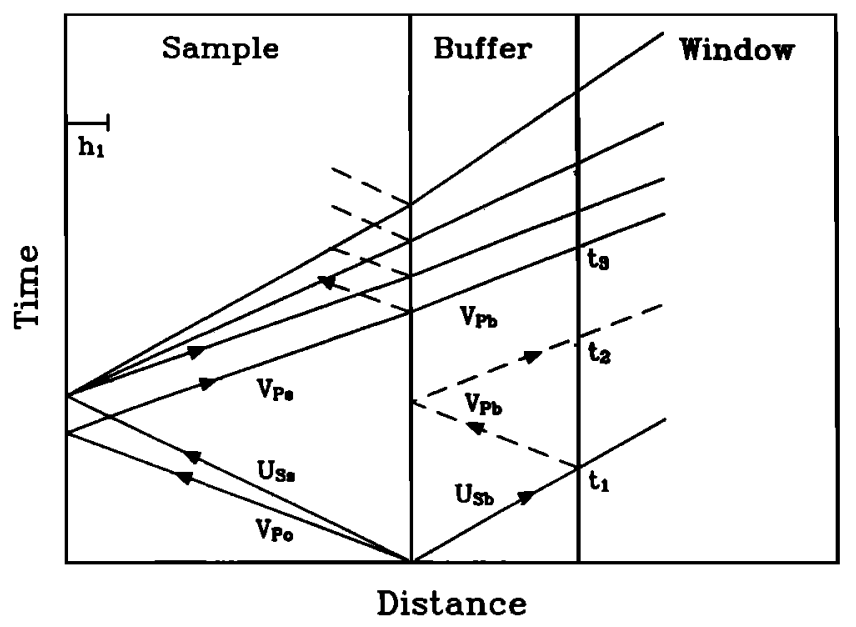

Figure 1b. Lagrangian distance-time diagram for reverse-impact experiments. The sample-foam boundary is at left edge of figure. $V_{P 0}$ is elastic precursor velocity, $U_{S}$ is shock velocity, $V_{P}$ is compressional sound velocity, $t_{1}-t_{3}$ are wave arrival times. $h_{1}$ is the point where reflected precursor and direct shock interact. The subscript $b$ and $s$ refer to the buffer and to the sample.

ther unloads the material. The unloading of the sample is recorded as a decrease in particle velocity beginning at $t_{3}$.

The forward impact experimental setup is shown in Figure 2a. In this case, the flyer plate impacts a sample to which a thin aluminum buffer has been epoxied. Sample-buffer and buffer-window epoxy layers were $\sim 10 \mu \mathrm{m}$ thick in all VISAR experiments. A Lagrangian distance-time diagram for this geometry is shown in Figure 2b. Symmetric impact results in an elastic precursor followed by a plastic shock propagating through both the $\mathrm{MgO}$ flyer and the $\mathrm{MgO}$ sample. The impedance contrast between the sample and buffer causes a partial unloading wave (dashed lines) to propagate back through the sample. The precursor and shock travel through the buffer and reach the buffer-window interface at $t_{1}$ and $t_{2}$. The impedance contrast between the buffer and window has been neglected in this analysis. The elastic precursor in the flyer eventually reaches the back surface and reflects from it. It interacts with the oncoming shock wave at position $h_{1}$ and later interacts with the buffer-sample reflection at $h_{2}$. It reaches the buffer-window surface at $t_{3}$ and produces a decrease in particle velocity. Subsequently, the unloading fan from

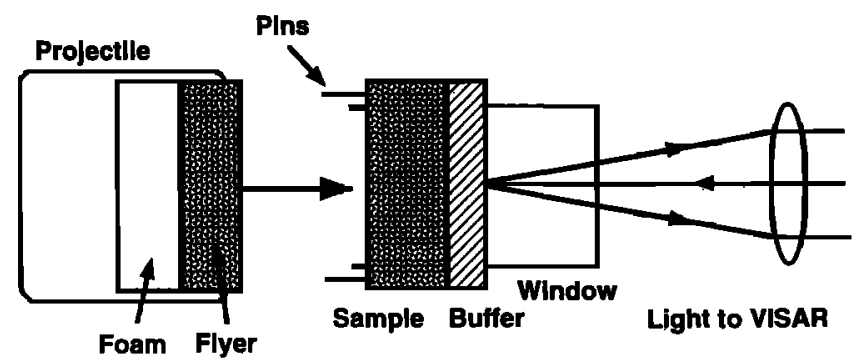

Figure 2a. Forward-impact experimental geometry.

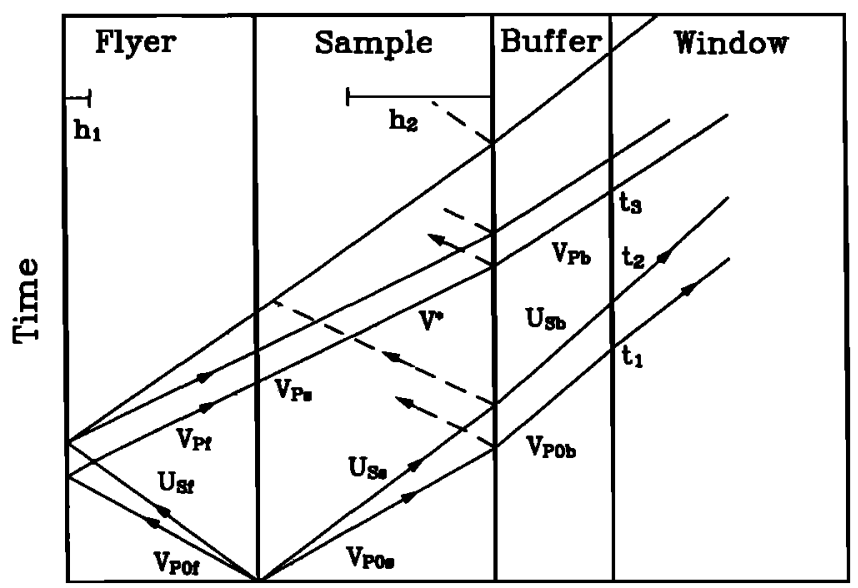

Distance

Figure 2b. Lagrangian distance-time diagram for forward impact experiments. The symbols are identified in Figure $1 \mathrm{~b} ; h_{2}$ is thickness of buffer-sample interaction region, and $V^{*}$ is the average velocity in the interaction region. The subscript $f$ refers to flyer properties.

the shock front arrives and further reduces the particle velocity.

The forward impacts have the disadvantage that the unloading portion of the waveform is significantly affected by wave interactions generated at the samplebuffer interface. These interactions are eliminated in the reverse geometry allowing determination of the unloading wave speed. The peak stress attainable in the reverse-impact arrangement using an $\mathrm{Al}$ buffer is limited to $\sim 27 \mathrm{GPa}$ with the present $40-\mathrm{mm}$ gun. The initial conditions for the VISAR experiments are listed in Tables 1 and 2.

\section{Results}

For the EOS experiments, the shock and flyer velocities were combined with impedance matching [Ahrens, 1987] and the Rankine-Hugoniot equations to constrain the particle velocity, stress, and density of the shockcompressed state (Table 3). The EOS properties of the flyer and driver plates are listed in Table 4. The elastic precursor was resolved in one experiment from which a precursor velocity of $9.77(12) \mathrm{km} / \mathrm{s}$ was obtained, which agrees with the ultrasonically measured sound velocity. The equation of state results are shown in Figures 3 and 4.

The buffer-window particle velocity histories measured in the reverse impacts are shown in Figure 5a. The sharp jump in particle velocity is the arrival of the shock front $\left(t_{1}\right)$. This is followed by the velocity plateau where some variations in particle velocity are evident. This may reflect differential motion of grains, material reorganization, or heterogeneous faulting and has been observed previously in velocity profile measurements on ceramics [Kipp and Grady, 1989]. Aluminum buffers were used to smooth out such irregularities. The arrival of the unloading waves produces a decrease in particle velocity starting at $t_{3}$ which displays an S-shaped struc- 
Table 1. Summary of Reverse-Impact Experiments

\begin{tabular}{|c|c|c|c|c|c|c|c|c|}
\hline \multirow[b]{2}{*}{ Shot } & \multicolumn{2}{|c|}{$\mathrm{MgO}$} & \multicolumn{2}{|c|}{ Al 6061 Buffer } & \multicolumn{2}{|c|}{ LiF Window } & \multirow{2}{*}{$\begin{array}{c}\text { Impact } \\
\text { Velocity, } \\
\text { km/s }\end{array}$} & \multirow{2}{*}{$\begin{array}{c}\text { Sample } \\
\text { Peak Stress } \\
\text { GPa }\end{array}$} \\
\hline & $\begin{array}{c}\rho_{0}, \\
\mathrm{~g} / \mathrm{cm}^{3}\end{array}$ & $\begin{array}{c}\text { Thickness, } \\
\text { mm }\end{array}$ & $\begin{array}{c}\rho_{0}, \\
\mathrm{~g} / \mathrm{cm}^{3}\end{array}$ & $\begin{array}{c}\text { Thickness, } \\
\text { mm }\end{array}$ & $\begin{array}{c}\rho_{0}, \\
\mathrm{~g} / \mathrm{cm}^{3}\end{array}$ & $\begin{array}{c}\text { Thickness, } \\
\text { mm }\end{array}$ & & \\
\hline 848 & $3.561(2)$ & $4.025(2)$ & $2.681(4)$ & $1.955(2)$ & $2.631(1)$ & $12.133(3)$ & $1.432(10)$ & $15.2(2)$ \\
\hline 851 & $3.569(8)$ & $3.032(6)$ & $2.681(3)$ & $1.962(2)$ & $2.630(1)$ & $12.138(2)$ & $2.366(35)$ & 27.4(6) \\
\hline 853 & $3.558(7)$ & $3.030(2)$ & $2.684(3)$ & $1.887(2)$ & $2.629(4)$ & $8.128(3)$ & $1.612(19)$ & $17.4(3)$ \\
\hline 854 & $3.568(3)$ & $4.018(2)$ & $2.687(5)$ & $1.880(3)$ & $2.626(1)$ & $12.142(2)$ & $1.020(9)$ & $10.4(2)$ \\
\hline 856 & $3.570(4)$ & $4.025(4)$ & $2.685(6)$ & $1.953(4)$ & $2.628(1)$ & 12.131(2) & $2.165(20)$ & $24.7(4)$ \\
\hline
\end{tabular}

Numbers in parentheses are one standard deviation uncertainties in the last digit(s).

ture. The final particle velocity level is dependent on the properties of the foam backing of the flyer.

The forward impact velocity histories are shown in Figure $5 \mathrm{~b}$. The first arrival is the elastic precursor $\left(t_{1}\right.$ in Figure 2b). The amplitude of this wave is a measure of the largest elastic stress that can be supported by the buffer under compressive loading. This is followed by the plastic shock front $\left(t_{2}\right)$ and the plateau region. Velocity variations in the plateau region are generally smaller for these experiments, probably because of the longer propagation distance. The shape of the unloading wave profile $\left(t_{3}\right)$ is similar to the reverse experiments, but the initial release is not as sharply defined in these experiments.

Hugoniot states were not obtained directly from the VISAR results as the impact times were not recorded. However, peak particle velocities from VISAR shots are consistent within experimental uncertainties with values from the $\mathrm{MgO}$ equation of state and impedance matching (see discussion section). The agreement between measured and calculated interface velocities can also be seen in the wavecode simulations discussed below. Calculated $\mathrm{MgO}$ longitudinal stresses achieved in the VISAR experiments are listed in Tables 1 and 2. The stress range covered by these experiments is 10-39 GPa.

Unloading wave velocities were determined from the reverse-impact experiments. For an elastic-plastic material, the initial unloading velocity is the compressional sound velocity $V_{P}$, and for a fluid it is the bulk velocity $V_{B}$ [Zel'dovich and Raizer, 1967]. The method for determining the unloading velocities can be seen with reference to Figure $1 \mathrm{~b}$. The time difference $\Delta t$ between the unloading wave arrival $t_{3}$ and the shock arrival $t_{1}$ is determined from the wave profile. The Lagrangian unloading velocity is given by

$$
V_{P L}=\frac{x_{s}-h_{1}}{\Delta t+x_{b} / U_{S b}-\left(x_{s}+h_{1}\right) / V_{P 0}-x_{b} / V_{P L b}},
$$

where $V_{P L}$ and $V_{P L b}$ are the Lagrangian sound velocities in the sample and buffer, $x_{s}$ and $x_{b}$ are the sample and buffer thicknesses, $U_{S b}$ is the buffer shock velocity, and $V_{P 0}$ is the elastic precursor velocity. $h_{1}$ is defined by the point of intersection of the elastic wave reflected from the rear surface of the flyer and the oncoming shock wave

$$
h_{1}=\frac{x_{s}\left(V_{P 0}-U_{S_{s}}\right)}{\left(V_{P 0}+U_{S_{s}}\right)}
$$

where $U_{S s}$ is the sample shock velocity. The ultrasonically determined compressional wave velocity was used for the precursor velocity. Shock states in the buffer and sample were determined from impedance matching using the data of Table 4.

Compressional velocities in the buffer were determined from the time difference between the shock arrival, $t_{1}$, and the arrival of the reverberation through the buffer, $t_{2}$ (Figure 1b) using

$$
V_{P L b}=\frac{2 x_{b}}{t_{2}-t_{1}} .
$$

Only experiments in which the reverberation arrival was clearly evident were used here. Lagrangian velocities

\begin{tabular}{|c|c|c|c|c|c|c|c|c|}
\hline \multirow[b]{2}{*}{ Shot } & \multicolumn{2}{|c|}{ Flyer } & \multicolumn{2}{|c|}{ MgO Sample } & \multicolumn{2}{|c|}{ Al 6061 Buffer } & \multirow{2}{*}{$\begin{array}{c}\text { Impact } \\
\text { Velocity, } \\
\text { km/s }\end{array}$} & \multirow{2}{*}{$\begin{array}{c}\text { Sample } \\
\text { Peak Stress, } \\
\text { GPa }\end{array}$} \\
\hline & $\begin{array}{c}\rho_{0}, \\
\mathrm{~g} / \mathrm{cm}^{3}\end{array}$ & $\begin{array}{c}\text { Thickness, } \\
\text { mm }\end{array}$ & $\begin{array}{c}\rho_{0}, \\
\mathrm{~g} / \mathrm{cm}^{3}\end{array}$ & $\begin{array}{c}\text { Thickness, } \\
\text { mm }\end{array}$ & $\begin{array}{c}\rho_{0}, \\
\mathrm{~g} / \mathrm{cm}^{3}\end{array}$ & $\begin{array}{c}\text { Thickness, } \\
\text { mm }\end{array}$ & & \\
\hline 857 & $3.563(4)$ & $3.024(2)$ & $3.561(4)$ & $4.030(4)$ & $2.683(3)$ & $1.944(2)$ & $1.520(20)$ & $21.4(3)$ \\
\hline 859 & $3.563(4)$ & $3.026(3)$ & $3.564(2)$ & $4.023(2)$ & $2.679(3)$ & $1.942(2)$ & $2.432(30)$ & $37.6(8)$ \\
\hline 861 & $3.558(4)$ & $3.030(2)$ & $3.551(6)$ & $4.025(2)$ & $2.684(3)$ & $1.980(2)$ & $1.015(8)$ & $13.5(2)$ \\
\hline 864 & $3.836(12)$ & 3.217(10) & $3.566(5)$ & $3.780(4)$ & $2.687(4)$ & $1.946(3)$ & $2.471(32)$ & $38.9(1.0)$ \\
\hline
\end{tabular}
were converted to Eulerian velocities $V_{P}$ by multiply-

Table 2. Summary of Forward-Impact Experiments

Flyers were $\mathrm{MgO}$ except for shot 864 , which used $\mathrm{Al}_{2} \mathrm{O}_{3}$. $\mathrm{LiF}$ windows had nominal density of $2.63 \mathrm{~g} / \mathrm{cm}^{3}$ and thickness of $8.15 \mathrm{~mm}$. Numbers in parentheses are one standard deviation uncertainties in the last digit(s). 
Table 3. Results of Equation of State Experiments

\begin{tabular}{lcccccccc}
\hline Shot & Flyer/Driver & $\begin{array}{c}u_{f p}, \\
\mathrm{~km} / \mathrm{s}\end{array}$ & $\begin{array}{c}\rho_{0}, \\
\mathrm{~g} / \mathrm{cm}^{3}\end{array}$ & $\begin{array}{c}u_{p}, \\
\mathrm{~km} / \mathrm{s}\end{array}$ & $\begin{array}{c}U_{S}, \\
\mathrm{~km} / \mathrm{s}\end{array}$ & $\begin{array}{c}\sigma, \\
\mathrm{GPa}\end{array}$ & $\begin{array}{c}\rho, \\
\mathrm{g} / \mathrm{cm}^{3}\end{array}$ & $\begin{array}{c}V_{P 0}, \\
\mathrm{~km} / \mathrm{s}\end{array}$ \\
\hline 233 & $\mathrm{Ta}$ & $4.876(3)$ & $3.566(12)$ & $3.367(12)$ & $11.042(73)$ & $132.6(8)$ & $5.130(27)$ & - \\
840 & $\mathrm{Ta}$ & $1.291(11)$ & $3.552(10)$ & $0.893(8)$ & $7.96(11)$ & $25.3(3)$ & $4.001(15)$ & - \\
841 & $\mathrm{Ta}$ & $2.521(30)$ & $3.561(6)$ & $1.742(23)$ & $9.01(13)$ & $55.9(9)$ & $4.415(25)$ & - \\
843 & $\mathrm{Mg} / \mathrm{Al} 2024$ & $1.713(17)$ & $3.566(12)$ & $\mathbf{0 . 5 1 3 ( 1 0 )}$ & $7.53(12)$ & $13.8(2)$ & $3.827(16)$ & $9.77(12)$ \\
\hline
\end{tabular}

Here $u_{f p}$ is the impact velocity, $u_{p}$ is the particle velocity, $U_{S}$ is the shock velocity, $V_{P 0}$ is the elastic precursor velocity, and the subscript zero refers to ambient pressure conditions. Numbers in parentheses are one standard deviation uncertainties in the last digit(s).

ing by the ratio of densities $\rho_{0} / \rho$. The Eulerian compressional velocities determined for the buffer material, Al 6061, are shown in Figure 6. Also shown are some Al 6061 sound velocities determined in separate experiments (T. S. Duffy, unpublished data) as well as data of Asay and Chhabildas [1981] for this material. The variation of $V_{P}$ with Hugoniot stress in $\mathrm{Al} 6061$ (Figure 6) can be described by

$$
\ln V_{P}=1.8796+0.0303 \ln \sigma+0.0177 \ln ^{2} \sigma,
$$

where $V_{P}$ is in $\mathrm{km} / \mathrm{s}$ and $\sigma$ is in GPa. The Eulerian unloading velocities in $\mathrm{MgO}$ determined from (2) are shown in Figure 7 where the axial stress has been converted to mean pressure. Uncertainties in the velocities are typically $\pm 2 \%$.

\section{Discussion}

\section{Equation of state}

While single-crystal and porous polycrystalline $\mathrm{MgO}$ have been studied extensively using shock techniques, the present study represents the first EOS determination for low-porosity (<1\%) polycrystalline MgO. A least squares fit to the single-crystal $\mathrm{MgO}$ data listed by Marsh [1980] yields (Figure 3)

$$
U_{S}=6.61(5)+1.36(2) u_{p} .
$$

The intercept of the $U_{S}-u_{p}$ relation corresponds to the bulk sound velocity for a material undergoing no high- pressure phase change. For $\mathrm{MgO}$, the bulk velocity from ultrasonic data [Jackson and Niesler, 1982] is 6.73(1) $\mathrm{km} / \mathrm{s}$, which is $1.9 \%$ above the $U_{S}-u_{p}$ intercept.

There is considerable evidence that single-crystal $\mathrm{MgO}$ has little or no strength when shocked above its elastic limit of $2.5 \mathrm{GPa}$. The Hugoniot stress-density states are nearly coincident with a $300 \mathrm{~K}$ hydrostatic compression curve at low stresses (Figure 4). The static isotherm was computed using ultrasonic elasticity data [Jackson and Niesler, 1982] corrected to isothermal conditions and extrapolated using the Birch-Murnaghan equation. Collapse to the hydrostat is also supported by features observed in wave profiles recorded for singlecrystal $\mathrm{MgO}$ at stresses of 5-11 GPa [Grady, 1977]. These include stress relaxation behind the precursor, shock velocities below the local bulk sound speed, and inferred stress-density states coincident with the hydrostat. These features are characteristic of strength collapse in brittle single crystals [Mashimo, 1993].

A least squares fit to the Hugoniot data for polycrystalline MgO yields (Figure 3)

$$
U_{S}=6.87(10)+1.24(4) u_{p},
$$

which is significantly different from the fit to the singlecrystal data. The intercept here lies above the ultrasonic bulk sound speed, which is commonly observed in materials with significant shear strength along the Hugoniot [McQueen et al., 1970]. Comparison with the $300 \mathrm{~K}$ hydrostat shows that Hugoniot states for polycrystalline $\mathrm{MgO}$ lie above the hydrostat at low stresses.

Table 4. Equation of State Standards

\begin{tabular}{lccccccr}
\hline Material & $\begin{array}{c}\rho_{0}, \\
\mathrm{~g} / \mathrm{cm}^{3}\end{array}$ & $\begin{array}{c}c_{0}, \\
\mathrm{~km} / \mathrm{s}\end{array}$ & $s$ & $\gamma_{0}$ & $\nu_{0}$ & $\begin{array}{c}Y, \\
\text { GPa }\end{array}$ & $\begin{array}{r}\text { Refer- } \\
\text { ences }\end{array}$ \\
\hline $\mathrm{MgO}$ & $3.562(6)$ & $6.87(10)$ & $1.24(4)$ & 1.52 & 0.18 & 1.25 & 1 \\
$\mathrm{Al} 6061$ & $2.683(3)$ & $5.349(56)$ & $1.338(20)$ & 2.10 & 0.34 & 0.3 & 2 \\
$\mathrm{Ta}$ & $16.65(3)$ & $3.293(5)$ & $1.307(25)$ & 1.60 & 0.34 & 0.75 & 3 \\
$\mathrm{LiF}$ & $2.64(2)$ & $5.15(3)$ & $1.35(1)$ & 1.63 & 0.22 & 0.2 & 2 \\
$\mathrm{Mg} \mathrm{AZ31B}$ & $1.775(1)$ & $4.52(5)$ & $1.26(2)$ & 1.43 & 0.30 & - & 2 \\
$\mathrm{Al}_{2} \mathrm{O}_{3}$ & $3.83(1)$ & $6.91(8)$ & $1.44(4)$ & 1.27 & 0.24 & 5.8 & 2 \\
\hline
\end{tabular}

Here $c_{0}$ and $s$ are Hugoniot equation of state constants, $\gamma_{0}$ is the Grüneisen parameter, $\nu_{0}$ is Poisson's ratio, and $Y$ is the yield strength. The references for Hugoniot properties are 1, this study; 2, Marsh [1980]; and 3, Mitchell and Nellis [1981]. The numbers in parentheses are one standard deviation uncertainties in the last digit(s). 


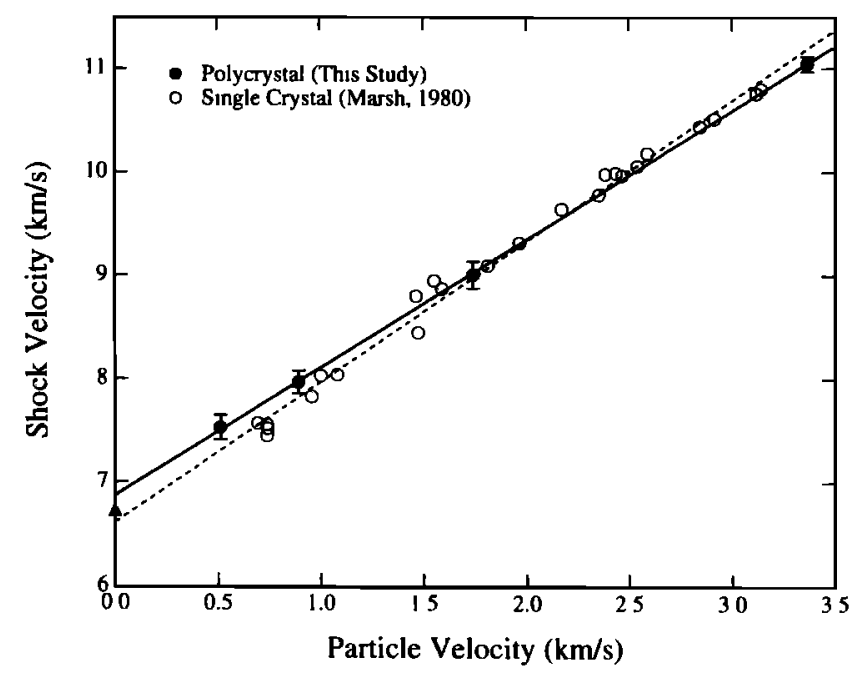

Figure 3. Shock velocity-particle velocity data for MgO. Solid symbols are data of this study. The triangle is bulk sound velocity from ultrasonic data. Solid curve is a least squares fit to polycrystalline data, and dashed curve is a fit to single-crystal data.

An estimate of the shear strength can be made from the stress difference between the Hugoniot and isotherm after thermal and porosity effects have been subtracted using the Mie-Grüneisen equation. For the three lowest stress data, the average corrected stress difference between the Hugoniot and isotherm is $1.8(5) \mathrm{GPa}$. The yield strength, $Y$, can then be determined using

$$
Y=\frac{3}{2}(\sigma-P),
$$

where $\sigma$ is the axial stress and $P$ is the pressure.

For polycrystalline $\mathrm{MgO}$, this yields $Y=2.7(8) \mathrm{GPa}$ at stresses of 14-56 GPa. Thus the EOS data reveal significant differences in the behavior of single-crystal

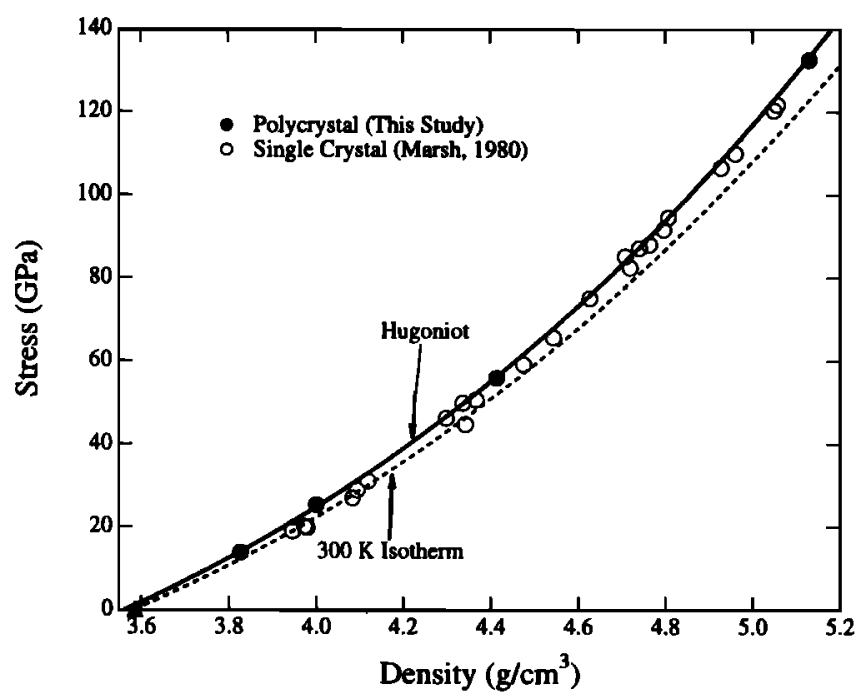

Figure 4. Stress-density states in shock compressed MgO. The ambient pressure density is shown by the triangle. The solid curve is the polycrystalline Hugoniot, and the dashed curve is the $300 \mathrm{~K}$ isotherm computed from extrapolated ultrasonic data.
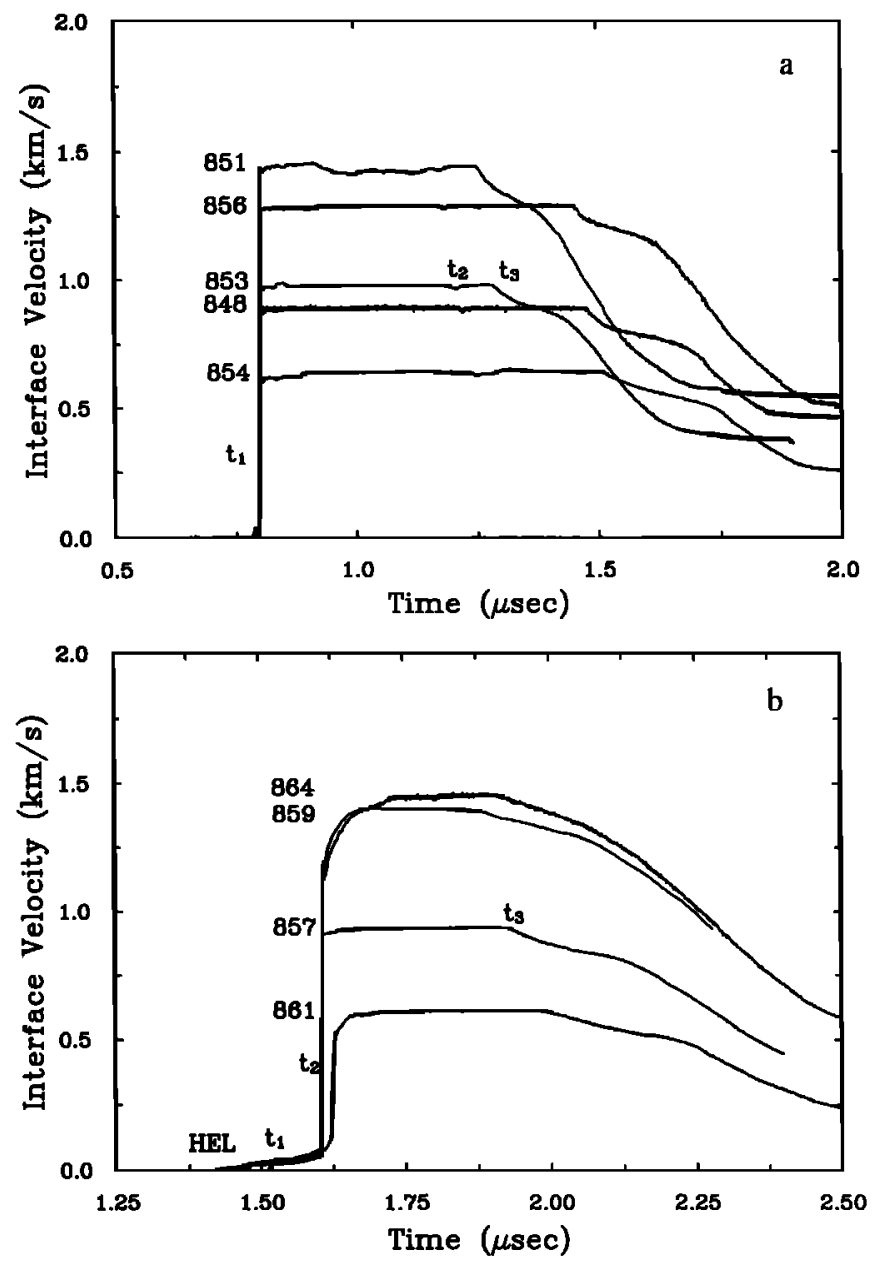

Figure 5. Interface particle velocity histories for (a) reverse and (b) forward impact experiments. Shot numbers are listed next to each profile. Times $t_{1}-t_{3}$ correspond to those in the $x$-t diagrams of Figures 1 and 2. Starting times are arbitrary. The elastic precursor is labeled HEL.

and polycrystalline $\mathrm{MgO}$. Similar differences have been observed in the shock response of other oxides such as $\mathrm{Al}_{2} \mathrm{O}_{3}$ and $\mathrm{ZrO}_{2}$ [Grady, 1977; Mashimo, 1993].

It is of interest to compare the dynamic yield strength of $\mathrm{MgO}$ with recent static measurements in the diamond anvil cell and multi-anvil press. Meade and Jeanloz [1988] measured static shear stresses in the range of 1-4 GPa at pressures of 10-40 GPa in diamond anvil cell experiments on $\mathrm{MgO}$. More recently, measurements of the static strength of $\mathrm{MgO}$ to $227 \mathrm{GPa}$ have shown that the strength is at least $11 \mathrm{GPa}$ at that pressure [Duffy et al., submitted manuscript]. Microscopic yield strengths of 2-4 GPa were obtained in multi-anvil experiments on $\mathrm{MgO}$ to $500^{\circ} \mathrm{C}$ at $8 \mathrm{GPa}$ [Weidner et al., 1994]. The dynamic yield strengths obtained here are similar to static values despite large differences in strain rates between static and dynamic experiments. Differences in the strength of single and polycrystals of $\mathrm{MgO}$ were also observed under static conditions [Meade and Jeanloz, 1988].

Reduction of the Hugoniot data to an adiabat was carried out using Mie-Grüneisen theory [Heinz and Jean- 


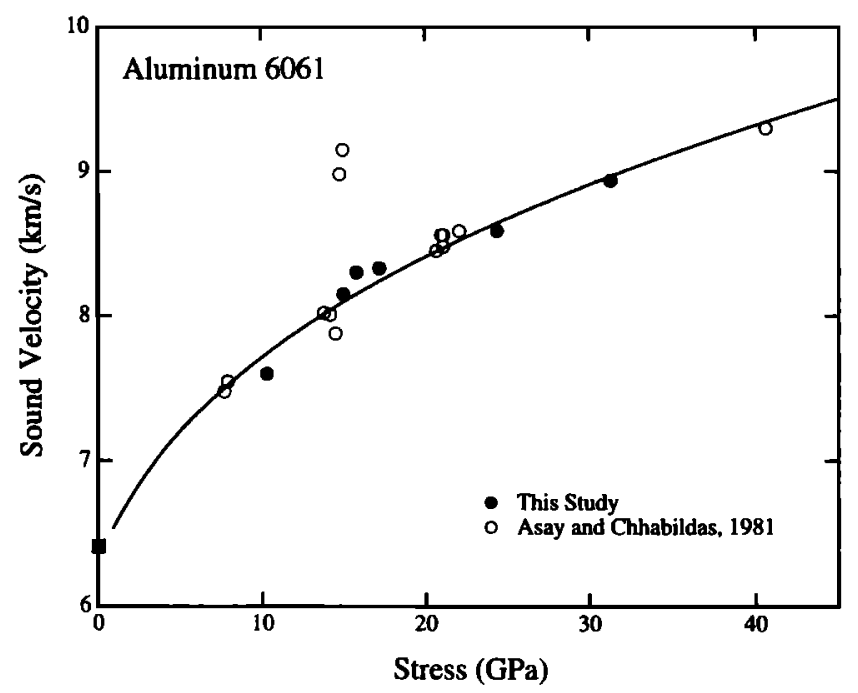

Figure 6. Compressional wave velocities in 6061 aluminum. The solid square is the ambient pressure ultrasonic velocity.

loz, 1984; Ahrens and Jeanloz, 1987]. The Grüneisen parameter $\gamma$ and the exponent specifying its volume dependence $q$ are listed in Table 5. The reduced Hugoniot data are displayed in a normalized pressure-strain representation [Birch, 1978] in Figure 8. In coordinates of Figure 8, the Eulerian finite strain equation of state is

$$
F=a_{0}+a_{1} f+a_{2} f^{2}+\ldots,
$$

where $a_{0}$ is the ambient pressure adiabatic bulk modulus, $K_{O S}$, and its first pressure derivative at constant entropy, $K_{0 S}^{\prime}=\left(\partial K_{0 S} / \partial P\right)_{S}$, and second pressure derivative at constant entropy, $K_{0 S}^{\prime \prime}=\left(\partial^{2} K_{0 S} / \partial P^{2}\right)_{S}$, appear

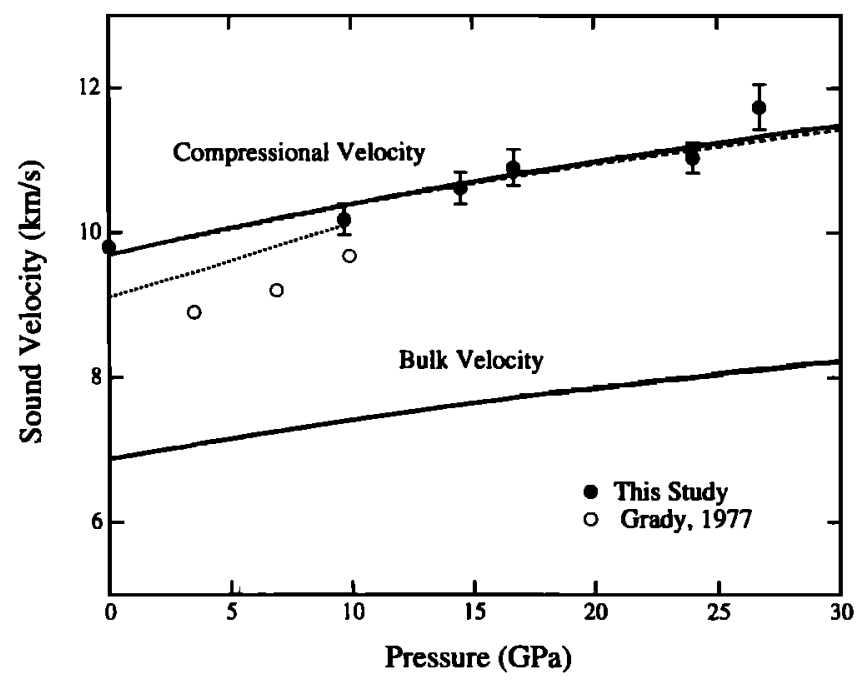

Figure 7. Compressional wave velocities in $\mathrm{MgO}$. Solid symbols are polycrystalline $\mathrm{MgO}$, and open symobls are single-crystal MgO shocked along [100]. Solid curves are third-order finite strain extrapolations of ultrasonic data at $300 \mathrm{~K}$ for $V_{P}$ and $V_{B}$, using parameters of Table 5. Long dashed curve shows maximum expected effect of Hugoniot temperature on $V_{P}$. Short dashed curve is extrapolated compressional velocity along [100].
Table 5. Elastic and Thermodynamic Properties of $\mathrm{MgO}$

\begin{tabular}{lcc}
\hline \multicolumn{1}{c}{ Property } & Value & Reference \\
\hline$\rho_{0}, \mathrm{~g} / \mathrm{cm}^{3}$ & $3.584(1)$ & 1 \\
$K_{0 S}, \mathrm{GPa}$ & $162.5(2)$ & 1 \\
$\left(\partial K_{0 S} / \partial P\right)_{S}$ & $4.09(9)$ & 1 \\
$\left(\partial^{2} K_{0 S} / \partial P^{2}\right)_{S}, \mathrm{GPa}^{-1}$ & $-0.019(4)$ & 3 \\
$G_{0}, \mathrm{GPa}$ & $130.8(2)$ & 1 \\
$\partial G_{0} / \partial P$ & $2.5(1)$ & 1 \\
$\partial^{2} G_{0} / \partial P^{2}, \mathrm{GPa}^{-1}$ & $-0.026(45)$ & 3 \\
$\gamma_{0}$ & $1.52(2)$ & 2 \\
$q ; \gamma=\gamma_{0}\left(\rho_{0} / \rho\right)^{q}$ & $1.0(5)$ & 4 \\
\hline
\end{tabular}

References are 1, Jackson and Niesler [1982]: 2, Sumino et al. [1983]; 3, this study; and 4, assumed. Numbers in parentheses are one standard deviation uncertainties in the last digit(s).

in the $a_{1}$ and $a_{2}$ terms, respectively. $f$ is the Eulerian strain given by

$$
f=\frac{1}{2}\left[\left(\frac{\rho}{\rho_{0}}\right)^{2 / 3}-1\right] .
$$

The normalized pressure $F$ is

$$
F=\frac{P}{3 f(1+2 f)^{5 / 2}} .
$$

Truncation of (9) after the linear strain term yields the third-order Birch-Murnaghan equation, while inclusion of the quadratic term gives the fourth-order form.

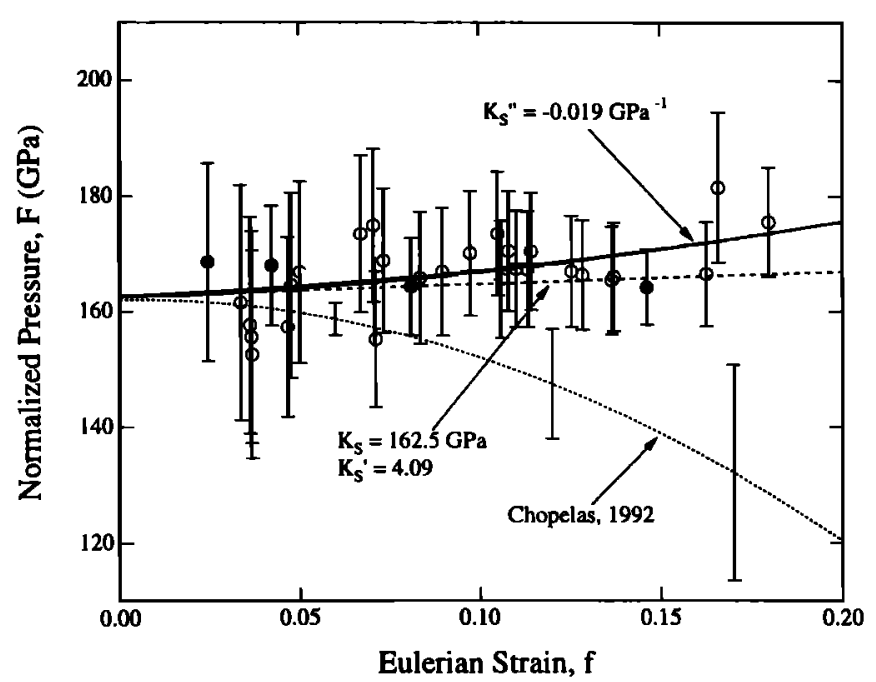

Figure 8. Hugoniot EOS data reduced to a principal isentrope and plotted in normalized pressure-strain coordinates. Solid symbols are polycrystalline data and open symbols are single-crystal data. Long dashed curve shows 3-GPa ultrasonic data extrapolated using a third-order Birch-Murnaghan equation. Solid curve is a fourth-order Birch-Murnaghan fit to shock data with $K_{0 S}$ and $K_{0 S}^{\prime}$ constrained at ultrasonic values. Shortdashed curve shows fourth-order Birch-Murnaghan fit using parameters of Chopelas [1992]. 
Analogous finite strain expressions can also be developed for the variation of sound velocity with strain [Sammis et al., 1970] as discussed below.

Ultrasonic sound velocity measurements have been made on $\mathrm{MgO}$ single crystals to $3 \mathrm{GPa}$ [Jackson and Niesler, 1982], which constrain the aggregate elastic moduli to $0.1 \%$ and their pressure derivatives to $\sim 4 \%$ (Table 5). A third-order $\left(a_{2}=0\right)$ finite strain extrapolation of these data is compared with the reduced Hugoniot data in Figure 8. A small correction has been made to convert the pressure derivative of the bulk modulus to constant entropy conditions. Both the reduced single-crystal and polycrystal Hugoniot data are consistent with extrapolated ultrasonic data. The study of Jackson and Niesler [1982] possesses significant advantages over earlier high-pressure ultrasonic experiments on MgO. In particular, Jackson and Niesler [1982] performed their measurements to significantly higher pressure ( $3 \mathrm{GPa}$ ) than other workers and made corrections for transducer bond shifts. The second pressure derivatives of the bulk and shear moduli required for the fourth-order finite strain expansion are not wellresolved in their data.

The ambient pressure adiabatic bulk modulus reported by Jackson and Niesler [1982] (162.5 GPa) is consistent with other ultrasonic and resonance studies of $\mathrm{MgO}$ [Sumino et al., 1983]. However, static compression studies have consistently reported a significantly higher bulk modulus for $\mathrm{MgO}\left(K_{T} \sim 180 \mathrm{GPa}\right)$ [e.g., Perez-Albuerne and Drickamer, 1965; Weaver et al., 1971]. A recent static compression study to pressures in excess of $200 \mathrm{GPa}$ has shown that the high bulk modulus is due to the static shear strength of $\mathrm{MgO}$ [Duffy et al., submitted manuscript]. This study also showed that when this strength is accounted for, a single equation of state can successfully describe the results of shock, ultrasonic, and static compression experiments for $\mathrm{MgO}$.

Since the single-crystal Hugoniot equation of state data do not show evidence for material strength, it is possible to combine the Hugoniot and ultrasonic data to constrain the fourth-order term in the BirchMurnaghan equation [Jeanloz et al., 1991]. The results of the constrained fourth-order fit are shown in Figure 8 and do not differ greatly from the third-order fit. The second pressure derivative of the bulk modulus $K_{0 S}^{\prime \prime}$ is determined to be $-0.019(4) \mathrm{GPa}^{-1}$. In contrast, Chopelas [1992] obtained $\left(\partial^{2} K_{0 S} / \partial P^{2}\right)_{T}=$ $-0.054(16) \mathrm{GPa}^{-1}$ from fluorescence sideband measurements on $\mathrm{MgO}$ to $20 \mathrm{GPa}$ at room temperature. The second derivative of Chopelas [1992] is not consistent with high-pressure Hugoniot data (Figure 8). The pressure derivatives of Chopelas [1992] have been corrected to constant entropy conditions $\left(K_{0 S}^{\prime}=4.04, K_{0 S}^{\prime \prime}=\right.$ $\left.-0.052 \mathrm{GPa}^{-1}\right)$ in making this comparison.

The elastic properties and equation of state of $\mathrm{MgO}$ have been calculated to $150 \mathrm{GPa}$ using an ab initio potential-induced breathing (PIB) model [Isaak et al., 1990]. The second pressure derivative of the isothermal bulk modulus at constant temperature, $\left(\partial^{2} K_{0 T} / \partial P^{2}\right)_{T}$, found in that study is $-0.026 \mathrm{GPa}^{-1}$ and the derivative of the adiabatic modulus is, $\left(\partial^{2} K_{0 S} / \partial P^{2}\right)_{T}=$
-0.023 $\mathrm{GPa}^{-1}$. Bukowinski [1985], using the augmented plane wave (APW) method, obtained $\left(\partial^{2} K_{0 T} / \partial P^{2}\right)_{T}$ $=-0.030 \mathrm{GPa}^{-1}$ for $\mathrm{MgO}$. By including a correction term which accounts for the energy difference between the adiabat and the isotherm, isothermal moduli and derivatives can be extracted from reduced Hugoniot data [Heinz and Jeanloz, 1984]. For the single-crystal shock data, this gives $\left(\partial^{2} K_{0 T} / \partial P^{2}\right)_{T}=-0.022(4) \mathrm{GPa}^{-1}$, which is similar to values from first-principle calculations.

The value of $K_{0 S}^{\prime \prime}$ which results when the finite strain expression is truncated at third order is $-0.025(1) \mathrm{GPa}^{-1}$, which differs only slightly from the value found here using shock compression data $\left(-0.019(4) \mathrm{GPa}^{-1}\right)$. This indicates that the fourth-order term is at best only marginally required for describing $\mathrm{MgO}$, in agreement with the results of Jackson and Niesler [1982].

The value of the second pressure derivative resulting from the reduced shock data is sensitive to the choice of the logarithmic volume dependence of the Grüneisen parameter $q$ [Jeanloz, 1989]. The range of $q$ values considered here $(\mathbf{0 . 5}-1.5)$ is consistent with ambient pressure thermoelastic data [Isaak et al., 1989], theory [Isaak et al., 1990; Anderson et al., 1993], Hugoniot data for porous $\mathrm{MgO}$ [Carter et al., 1971], and shock temperature data [Svendsen and Ahrens, 1987]. In order for the reduced shock data to be consistent with the second pressure derivative of Chopelas [1992], it is necessary to have $q$ values of -1 to -3 . Such values are inconsistent with the above constraints and are physically implausible because they lead to negative sound velocities on the Hugoniot [McQueen et al., 1967; Jean$l o z, 1992]$. Furthermore, a room temperature equation of state constructed by correcting Chopelas's [1992] results to the isothermal moduli deviates strongly from measured pressure-volume data above $50 \mathrm{GPa}$ at $300 \mathrm{~K}$ [Duffy et al., submitted manuscript].

\section{Compressional Wave Velocity}

The compressional wave velocities measured under shock compression are compared to third-order finite strain extrapolation of ultrasonic data in Figure 7. The Hugoniot measurements agree with the ultrasonic extrapolations within their experimental precision. This supports the use of third-order finite strain theory for extrapolating compressional wave velocities in $\mathrm{MgO}$ over pressures nearly a factor of 10 greater than the experimental data and into the pressure range of the lower mantle. The dependence of compressional sound velocity on Hugoniot pressure in $\mathrm{MgO}$ to $27 \mathrm{GPa}$ is given by

$$
\ln V_{P}=2.2790-0.0624 \ln P+0.0344 \ln ^{2} P,
$$

where $V_{P}$ is in $\mathrm{km} / \mathrm{s}$ and $P$ is in GPa.

Hugoniot sound velocity measurements can be inverted to constrain elastic moduli and their pressure derivatives along the Hugoniot. In the region where thermal effects are small, these are comparable to ultrasonic determinations. By analogy to (9), the fourth- 
order finite strain expression for compressional velocity can be written [Duffy and Ahrens, 1992]

$$
\frac{\rho V_{P}^{2}}{(1+2 f)^{5 / 2}}=a_{L 0}+a_{L 1} f+a_{L 2} f^{2}
$$

where

$$
\begin{gathered}
a_{L 0}=K_{0 S}+4 / 3 G_{0} \\
a_{L 1}=3 K_{0 S}\left(K_{0 S}^{\prime}+4 / 3 G_{0}^{\prime}\right)-5\left(K_{0 S}+4 / 3 G_{0}\right), \\
a_{L 2}=\frac{9}{2} K_{0 S}^{2}\left(K_{0 S}^{\prime \prime}+4 / 3 G_{0}^{\prime \prime}\right)+\frac{9}{2} K_{0 S}\left(K_{0 S}^{\prime}-4\right) \\
\times\left(K_{0 S}^{\prime}+4 / 3 G_{0}^{\prime}\right)+\frac{35}{2}\left(K_{0 S}+\frac{4}{3} G_{0}\right),
\end{gathered}
$$

where $G$ is the shear modulus and primes represent first and second pressure derivatives, respectively. As with EOS data, the Hugoniot sound velocities were combined with ultrasonic moduli and first pressure derivatives to constrain the second pressure derivatives of the moduli. The fit yields $K_{0 S}^{\prime \prime}+4 / 3 G_{0}^{\prime \prime}=-0.053(60) \mathrm{GPa}^{-1}$. Together with the value of $K_{0 S}^{\prime \prime}=-0.19(4) \mathrm{GPa}^{-1}$ determined above, this implies that $G_{0}^{\prime \prime}=-0.026(45) \mathrm{GPa}^{-1}$. The elastic properties of $\mathrm{MgO}$ are listed in Table 5. The PIB model calculations yield $G_{0}^{\prime \prime}=-0.020 \mathrm{GPa}^{-1}$. The fluorescence sideband measurements of Chopelas [1992] yield $G_{0}^{\prime \prime}=-0.034(10) \mathrm{GPa}^{-1}$.

Comparison of Hugoniot data with $300 \mathrm{~K}$ extrapolations of ultrasonic data can be biased because of thermal effects on the Hugoniot. Between 10 and $27 \mathrm{GPa}$, the continuum Hugoniot temperatures in $\mathrm{MgO}$ are calculated to lie between 320 and $408 \mathrm{~K}$ using the method of $M c Q u e e n$ et al. [1970]. From the ambient pressure value of the temperature coefficient of compressional velocity for MgO [Isaak et al., 1989], an upper bound to temperature correction can be determined (Figure 7). The thermal effect on the velocities is significantly less than experimental uncertainties.

It is also of interest to compare sound velocities in single-crystal and polycrystalline $\mathrm{MgO}$. Unloading wave velocities in single-crystal $\mathrm{MgO}$ shocked along [100] to 5 -11 GPa are $\sim 5 \%$ below expected values based on extrapolated ultrasonic data [Grady, 1977] (Figure 7). This suggests that the strength of the single-crystal material has substantially but not completely recovered in the 500-ns time interval between shock and release. The recovery may be related to the relatively large thermal diffusivity in single-crystal $\mathrm{MgO}$ which leads to rapid thermal equilibration [Grady, 1980]. Polycrystalline $\mathrm{MgO}$, on the other hand, initially unloads in a purely elastic fashion.

\section{Constitutive Behavior}

Numerical simulations of the particle velocity histories were carried out using the one-dimensional finite difference wave code WONDY [Kipp and Lawrence, 1982]. This program simulates plate impact and other experimental geometries by solving the equations of conservation of mass, momentum, and energy together with an appropriate constitutive law. Discontinuities associated with shock fronts are treated by the method of artificial viscosity. In general, the stress consists of a pressure (volumetric) term and a deviatoric term $\sigma^{\prime}$

$$
\sigma=P+\sigma^{\prime}
$$

where both stresses and pressures are taken to be positive in compression. The volumetric portion is described by the Mie-Grüneisen equation

$$
P=P_{H}+\frac{\gamma}{V}\left(E-E_{H}\right),
$$

where $V$ is the volume, $E$ is the energy, and the subscript $H$ refers to the reference Hugoniot state. The Grüneisen parameter is modeled assuming $q=1$ (Table 5) as

$$
\rho \gamma=\rho_{0} \gamma_{0} .
$$

The bulk sound velocity is given by

$$
V_{B}^{2}=\left(\frac{\partial P}{\partial \rho}\right)_{S},
$$

and the compressional sound velocity is

$$
V_{P}^{2}=3\left(\frac{1-\nu}{1+\nu}\right) V_{B}^{2}
$$

where $\nu$ is Poisson's ratio.

The stress deviators are obtained from a relation of the form

$$
\partial \sigma^{\prime} / \partial t=2 G(\partial \epsilon / \partial t-g),
$$

where $\epsilon$ is the engineering strain, $t$ is time, and $g$ is a relaxation function.

A simple description for solids is the elastic-perfectly plastic model. Such a material behaves elastically until its yield point, after which it deforms plastically, maintaining a constant offset of $2 Y / 3$ from the hydrostat. Upon unloading, the material again behaves elastically until it reaches a state of stress of $2 Y / 3$ below the hydrostat, after which it decompresses irreversibly to zero stress.

The yield strength at the Hugoniot elastic limit (HEL) (assuming a von Mises yield condition) can be obtained from

$$
Y=\frac{(1-2 \nu)}{(1-\nu)} \sigma_{H E L}
$$

The HEL amplitude of polycrystalline $\mathrm{MgO}$ was not directly measured in these experiments, but the compressive wave structure was recorded at the buffer-window interface in the forward impact experiments.

The HEL amplitudes were modeled using WONDY simulations and an elastic-perfectly plastic model for both $\mathrm{MgO}$ and $\mathrm{Al}$ 6061. The model parameters are listed in Table 4. The results of simulations for two forward impacts are shown in Figure 9. The yield strength of Al 6061 was fixed at $0.3 \mathrm{GPa}$ [Asay and Lipkin, 1978]. The yield strength of polycrystalline $\mathrm{MgO}$ was varied between 0.5 and $2.5 \mathrm{GPa}$, and the predicted compressive waveforms are shown in Figure 9. For yield stresses above about $1.5 \mathrm{GPa}$, significant secondary structure is evident due to the onset of plastic deformation in the $\mathrm{Al}$ buffer. No such secondary structure is observed in the measured wave profiles. For yield stresses be- 


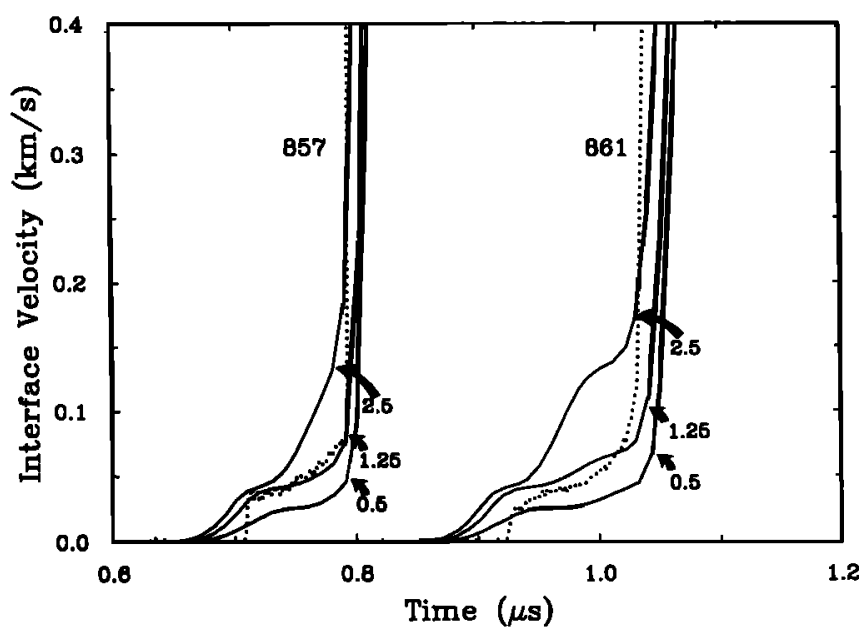

Figure 9. Comparison of compressive wave structure transmitted through aluminum buffer with wave code simulations for two forward impacts. Dotted curves are data (shot numbers shown next to each record). Solid curves are simulations using different values for the yield strength of polycrystalline $\mathrm{MgO}$. Computed profiles for yield strengths of $0.5,1.25$, and $2.5 \mathrm{GPa}$ are shown.

low 1.0 GPa, the predicted amplitude of the HEL lies well below the experimentally observed value. Yield stresses in the range of 1.0-1.5 GPa yield HEL amplitudes that are reasonably consistent $( \pm 10 \mathrm{~m} / \mathrm{s})$ with experimental values. We therefore adopt $Y=1.25(25)$ $\mathrm{GPa}$ as representing the possible range of compressive yield strengths of polycrystalline $\mathrm{MgO}$. This implies a Hugoniot elastic limit of 1.6(3) GPa. The HEL of singlecrystal $\mathrm{MgO}$ shocked along [100] was found to be 2.5 $\mathrm{GPa}$ for 3.3-mm-thick samples at peak stresses between 4.8 and $11.2 \mathrm{GPa}$ [Grady, 1977]. Ahrens [1966] observed elastic wave amplitudes of 3.5-8.9 GPa in single-crystal $\mathrm{MgO}$ shocked to peak stresses between 16.6 and 42.3 $\mathrm{GPa}$. The precursor amplitude in the polycrystalline material is significantly less than single-crystal values. This has been observed before in other materials, notably $\mathrm{Al}_{2} \mathrm{O}_{3}$ [Graham and Brooks, 1971; Mashimo et al., $1988]$ and is a reflection of different yielding processes in single and polycrystals.

Wave profile and equation of state data for ceramics (e.g., $\mathrm{SiC}, \mathrm{B}_{4} \mathrm{C}, \mathrm{TiO}_{2}, \mathrm{ZrO}_{2}$, and $\mathrm{Al}_{2} \mathrm{O}_{3}$ ) suggest quite varied material response. Some materials $\left(\mathrm{SiC}, \mathrm{Al}_{2} \mathrm{O}_{3}\right)$ are consistent with relatively simple material models such as a modified elastic plastic model [Mashimo et al., 1988; Kipp and Grady, 1989]. Other materials exhibit anomalous dispersion that implies significant deviation from elastic-plastic behavior [Kipp and Grady, 1989].

In order to assess whether an elastic-plastic model is appropriate for magnesium oxide, numerical simulations of the experiments were undertaken utilizing an elastic-perfectly plastic (EPP) model for MgO. Al 6061 and $\mathrm{LiF}$ were also modeled as EPP with the parameters listed in Table 4. The foam model of Grady and Furnish [1988] was used to describe the flyer-backing material. The predicted particle velocity histories are compared to measured values for a representative reverse impact in Figure 10. The yield strength of $\mathrm{MgO}$ was assumed to be $1.25 \mathrm{GPa}$ in the first simulation (Figure 10a) and $2.5 \mathrm{GPa}$ in the second (Figure 10b). The EPP model with $Y=1.25 \mathrm{GPa}$ does a poor job of matching the observed wave profile. While the initial elastic release is well modeled, the bulk of the unloading history arrives too late in the simulation. This indicates that the unloading behavior of $\mathrm{MgO}$ is not significantly dispersive.

Much better agreement between calculation and experiment is obtained when the initial yield strength is $2.5 \mathrm{GPa}$ (Figure 10b). This figure shows that an elasticplastic model is generally appropriate for describing the dynamic response of $\mathrm{MgO}$. A detailed comparison of measured and calculated wave profiles indicates that these are some important deviations from the model, however. The initial yield strength is a factor of 2 larger than that inferred from the HEL amplitude. This implies that $\mathrm{MgO}$ undergoes significant strain hardening.

The EPP model predicts several distinct wave arrivals which are not observed. In order to improve agreement, we incorporated modifications to the elastic-perfectly
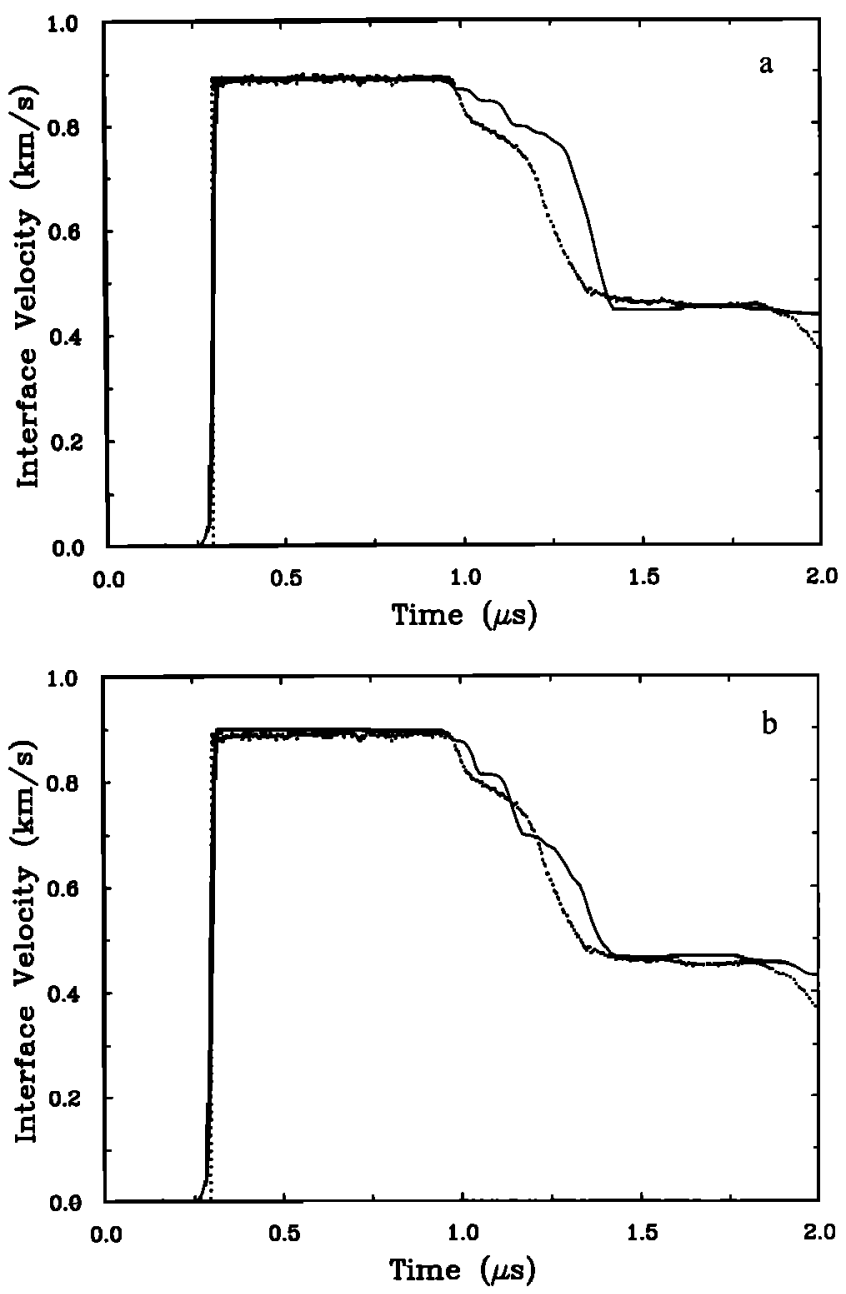

Figure 10. Calculated and measured particle velocity histories at the aluminum-LiF interface for experiment 848. Dotted curve is experimental profile, and solid curve is numerical simulation. $\mathrm{MgO}, \mathrm{Al}$, and $\mathrm{LiF}$ are treated as elastic-perfectly plastic. Yield strength of $\mathrm{MgO}$ is (a) $1.25 \mathrm{GPa}$ and (b) $2.5 \mathrm{GPa}$. 
plastic model that have been successfully applied to metals. In particular, we included a Bauschinger effect and strain rate dependent stress relaxation. In the Bauschinger effect, the yield stress is reduced when the direction of plastic deformation is reversed. This behavior is a consequence of the micromechanics of deformation, such as dislocation interaction, slip bands, and twinning. Flow stress anisotropy is implemented into the wavecode using a multielement kinematical model [Herrmann, 1974] in which the equilibrium stress deviator, $\sigma_{e}^{\prime}$, is given by

$$
\sigma_{e}^{\prime}=\sum a_{i} \sigma_{i}^{\prime}
$$

where the $a_{i}$ are normalized weighting factors. Each elemental stress deviator $\sigma_{i}^{\prime}$ is subject to a von Mises yield condition

$$
\sigma_{i}^{\prime 2} \leq\left(2 / 3 Y_{i}\right)^{2} .
$$

Strain rate dependence is also treated through the deviatoric stress. The stress deviator is obtained by relaxation from some instantaneous value to an equilibrium value, using the relaxation function in (22). The relaxation function has the form

$$
g=\frac{\sigma^{\prime}-\sigma_{e}^{\prime}}{G t_{r}}
$$

where $t_{r}$ is a characteristic material relaxation time.

Beginning with the elastic-perfectly plastic model, the wave profiles were fit iteratively by adjusting the parameters of the Bauschinger model and the relaxation time constant. For Al 6061, the Bauschinger model of Lawrence and Asay [1979] was used. Very little rate dependence is required in fitting the profiles. A time constant of $\mathbf{5}$ ns was found to improve the fit slightly for approximately the final $20 \%$ of the unloading history. The Bauschinger effect, on the other hand, is significant in $\mathrm{MgO}$.

We were unable to fit all of the data with a single model. The reason for this can be seen with reference to Figure 5. The reverse impacts exhibit significantly stronger and sharper initial release behavior than the forward experiments. The forward impacts involved a propagation distance through $\mathrm{MgO}$ of about $7 \mathrm{~mm}$, while in the reverse experiments the $\mathrm{MgO}$ propagation distance was 3-4 mm. This may indicate that the wave profiles are not steady. A second possibility is that the partial release generated at the sample-buffer interface in the forward experiments produces damage or defects which affect subsequent unloading waves. The lowestamplitude reverse impact (854) is similar to the forward impacts in that it has a weak and diffuse elastic release. In the forward impact experiments, the two higheststress experiments (859 and 864 ) show nearly featureless unloading, particularly shot 864 . These points illustrate that the shape of the profile in $\mathrm{MgO}$ is dependent both on peak stress and impact geometry.

The modeling procedure converged on two models for $\mathrm{MgO}$. The reverse impacts were fit using model 1 (Ta-

\begin{tabular}{|c|c|c|c|c|c|}
\hline \multicolumn{4}{|c|}{$\mathrm{MgO}$} & \multicolumn{2}{|c|}{ Al 6061} \\
\hline \multicolumn{2}{|c|}{ Model 1} & \multicolumn{2}{|c|}{ Model 2} & & \\
\hline$a_{i}$ & $Y_{i}, \mathrm{GPa}$ & $a_{i}$ & $Y_{i}, \mathrm{GPa}$ & $a_{i}$ & $Y_{i}, \mathrm{GPa}$ \\
\hline 0.7 & 1.5 & 0.10 & 2.95 & 0.581 & 0.169 \\
\hline 0.12 & 4.0 & 0.20 & 3.0 & 0.190 & 0.350 \\
\hline 0.10 & 8.0 & 0.20 & 3.1 & 0.078 & 0.472 \\
\hline 0.08 & 12.0 & 0.35 & 3.2 & 0.052 & 0.977 \\
\hline - & - & 0.09 & 3.5 & 0.063 & 1.649 \\
\hline - & - & 0.06 & 4.1 & 0.036 & 2.589 \\
\hline
\end{tabular}
ble 6) and are displayed in Figure 11a. This model
Table 6. Bauschinger Models for $\mathrm{MgO}$ and $\mathrm{Al} 6061$

reproduces the strong elastic release observed in these experiments. The primary difference between the model and the experimental records is that the model predicts a second elastic release originating from the reflection of the shock wave at the foam-flyer interface. In fact, the release at this point is highly dispersive.

Model 2 fits the forward impacts and the lowestamplitude reverse experiment. The Bauschinger parameters for this model are listed in Table 6, and the model predictions are compared to representative experimental data in Figure 11b. The agreement between data and calculation is good for the unloading portions of the waveform. The rise time of the shock front is longer in the model calculations than in the experimental data.

Figure 12 shows representative stress-strain histories at the center of the $\mathrm{MgO}$ samples for the forward and reverse impacts computed from the wave code simulations. Here the strain is defined as $\eta=1-\rho_{0} / \rho$. The Bauschinger effect is responsible for smoothing the transition from elastic to plastic strain upon both loading and unloading. This effect is much more pronounced in the forward impact model (model 2). The higherstress reverse-impact experiments (848 and 851 ) retain a clear transition from elastic to plastic unloading. The stress-strain states inferred from the wavecode simulations lie above the hydrostatic compression curve, as inferred from the equation of state experiments.

\section{Summary}

The Hugoniot of low-porosity $(0.5 \%)$ polycrystalline $\mathrm{MgO}$ between 14 and $133 \mathrm{GPa}$ is

$$
U_{S}=6.87(10)+1.24(4) u_{p},
$$

which differs significantly from that of single-crystal $\mathrm{MgO}$. Compressional sound velocities were determined from unloading wave profiles at $10-27 \mathrm{GPa}$. Within their $\pm 2 \%$ uncertainties, these data are consistent with ultrasonic data extrapolated from $3 \mathrm{GPa}$. This supports the use of third-order finite strain theory for comparing low-pressure laboratory measurements with seismological data to pressures of at least $\sim 25 \mathrm{GPa}$ for closepacked solids such as $\mathrm{MgO}$. By combining Hugoniot equation of state, Hugoniot sound velocity, and ultra- 

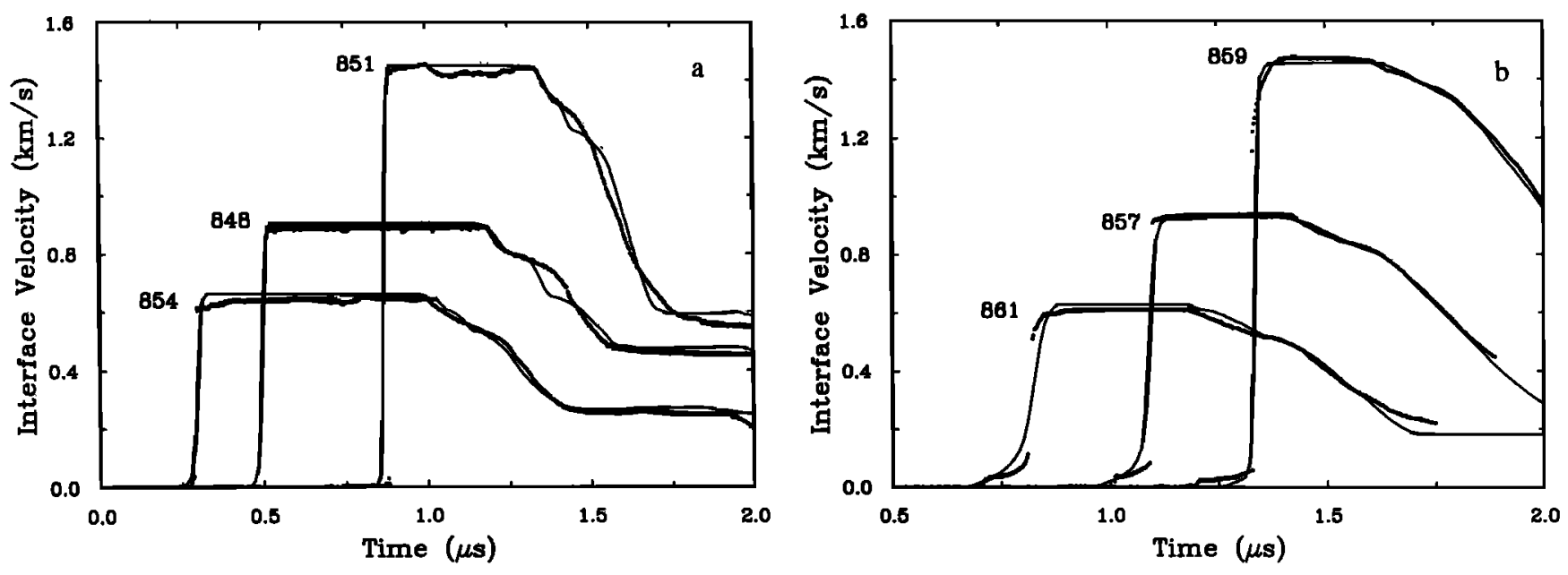

Figure 11. Numerical simulations of (a) reverse and (b) forward impacts. Symbols are experimental data, and solid curve is computed velocity history. Profile of shot 859 was scaled by 1.05 to bring its amplitude into agreement with calculated profile.

sonic data, the adiabatic bulk modulus, and its first and second pressure derivatives at constant entropy are 162.5(2) $\mathrm{GPa}, 4.09(9)$, and $-0.019(4) \mathrm{GPa}^{-1}$. The corresponding values for the aggregate shear modulus are 130.8(2) GPa, 2.5(1), and $-0.026(45) \mathrm{GPa}^{-1}$.

The dynamic response of $\mathrm{MgO}$ is strongly dependent on crystal state. Polycrystalline $\mathrm{MgO}$ is characterized by a low Hugoniot elastic limit, significant Hugoniot shear strength, work hardening, and fully elastic release. By comparison, single-crystal $\mathrm{MgO}$ has a large elastic limit, no strength along the Hugoniot, and quasi-elastic release [Grady, 1977]. However, it should be noted that wave profile data for the single crystal cover a lower stress range than the polycrystal data. Nevertheless, the differences seen here are similar of those observed in single-crystals and polycrystals of $\mathrm{Al}_{2} \mathrm{O}_{3}$ and $\mathrm{ZrO}_{2}$ [Mashimo, 1993]. We have demonstrated that such dif- ferences are also characteristic of the behavior of relatively low-strength ceramics like $\mathrm{MgO}$.

From wave profile and equation of state data, we infer that for polycrystalline $\mathrm{MgO}$, the yield strength at the HEL is $1-1.5 \mathrm{GPa}$, only $\sim 0.5$ the single-crystal value. The strength at the Hugoniot state is larger (2.8(7) $\mathrm{GPa}$ ) and this strength is largely maintained upon release. For single-crystals, the large strength (2.5 $\mathrm{GPa}$ ) at the HEL is reduced to near zero along the Hugoniot, but the strength is partially recovered during release (with a reduced shear modulus). Thus the shock-compression process is complex for both forms of this material and each exhibits its own time dependent strength behavior. While there are differences due to experimental configuration, the polycrystalline $\mathrm{MgO}$ wave profiles are generally consistent with the behavior of an elastic-plastic material.
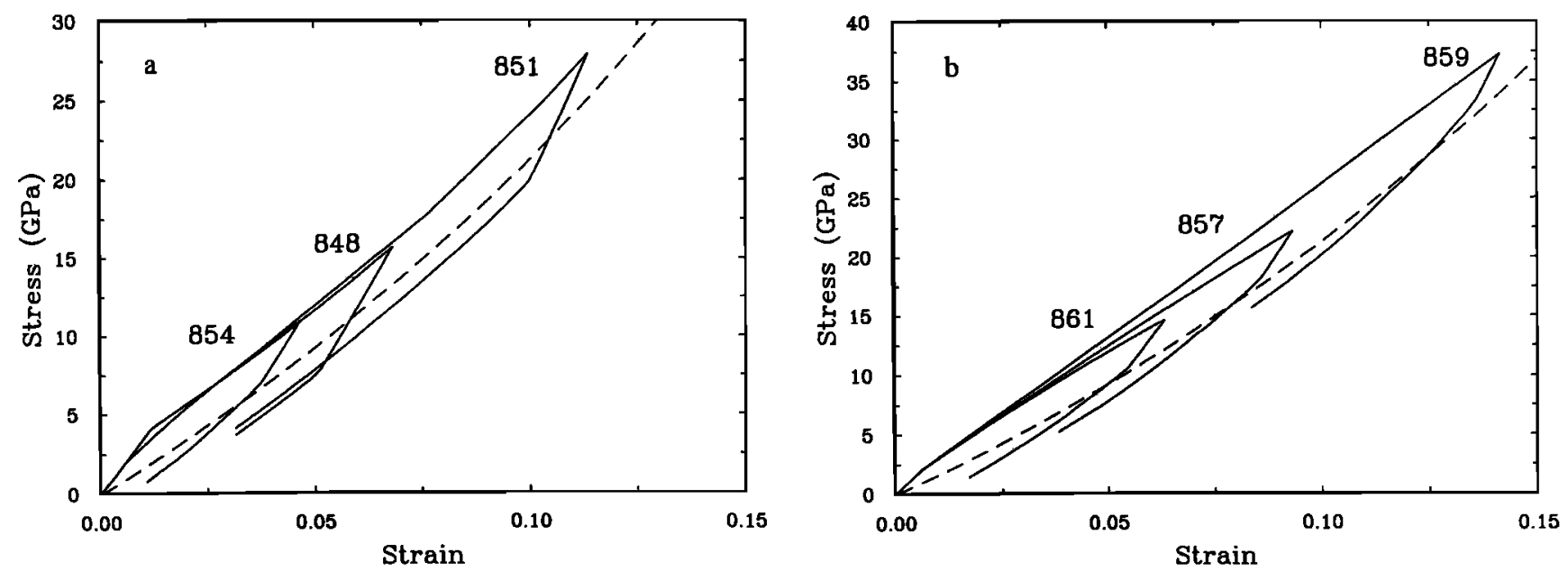

Figure 12. Stress-strain histories from numerical simulations of (a) reverse and (b) forward impact particle velocity histories of Figure 11. Dashed curve is the $300 \mathrm{~K}$ (hydrostatic) isctherm. 
Acknowledgments. We thank S. M. Rigden for performing the ultrasonic sound velocity measurements. $M$. Long, E. Gelle, and A. Devora provided experimental assistance. The comments of $R$. Jeanloz and two reviewers are appreciated. We also acknowledge $L$. Barker and O. B. Crump for helpful discussions. This research was supported by the NSF. Division of Geological and Planetary Sciences, California Institute of Technology contribution 5397.

\section{References}

Ahrens, T. J., High-pressure electrical behavior and equation of state of magnesium oxide from shock wave measurements, J. Appl. Phys., 37, 2532-2541, 1966.

Ahrens, T. J., Shock wave techniques for geophysics and planetary physics, in Methods of Experimental Physics, vol. 24, edited by C. G. Sammis and T. L. Henyey, pp. 185-235, Academic, San Diego, Calif., 1987.

Ahrens, T. J., and R. Jeanloz, Pyrite: Shock compression, isentropic release, and composition of the Earth's core, $J$. Geophys. Res, 92, 10,363-10,375, 1987.

Al'tshuler, L. V., S. B. Kormer, M. I. Brazhnik, L. A. Vladimirov, M. P. Speranskaya, and A. I. Funtikov, The isentropic compressibility of aluminum, copper, lead, and iron at high pressures, Sov. Phys. JETP, Engl. Transl., $11,761-775,1960$.

Anderson, O. L., H. Oda, A. Chopelas, and D. G. Isaak, A thermodynamic theory of the Grũneisen ratio at extreme conditions: $\mathrm{MgO}$ as an example, Phys. Chem. Mineral., $19,369-380,1993$.

Asay, J. R., and L. M. Barker, Interferometric measurement of shock-induced particle velocity and spatial variations of particle velocity, J. Appl. Phys., 45, 2540-2546, 1974.

Asay, J. R., and L. C. Chhabildas, Determination of the shear strength of shock compressed 6061-T6 aluminum, in Shock Waves and High Strain-Rate Phenomena in Metals, edited by M. A. Meyers, and L. E. Murr, pp. 417-431, Plenum, New York, 1981.

Asay, J. R., and J. Lipkin, A self-consistent technique for estimating the dynamic yield strength of a shock-loaded material, J. Appl. Phys., 49, 4242-4247, 1978.

Barker, L. M., and R. E. Hollenbach, Laser interferometer for measuring high velocities of any reflecting surface, $J$. Appl. Phys., 43, 4669-4675, 1972.

Birch, F., Finite strain isotherm and velocities for singlecrystal and polycrystalline $\mathrm{NaCl}$ at high pressures and 300 K, J. Geophys. Res., 83, 1257-1268, 1978.

Brown J. M., and R. G. McQueen, Phase transitions, Grüneisen parameter, and elasticity for shocked iron between $77 \mathrm{GPa}$ and $400 \mathrm{GPa}, J$. Geophys. Res., 91, 74857494, 1986.

Bukowinski, M. S. T., First principles equations of state of $\mathrm{MgO}$ and $\mathrm{CaO}$, Geophys. Res. Lett., 12, 536-539, 1985.

Carter, W. J., S. P. Marsh, J. N. Fritz, and R. G. McQueen, The equation of state of selected materials for high-pressure reference, in Accurate Characterization of the High-Pressure Environment, edited by E. C. Boyd, NBS Special Publ. 326, 147-158, 1971.

Chopelas, A., Sound velocities of MgO to very high compression, Earth Planet. Sci. Lett., 114, 185-192, 1992.

Duffy, T.S., and T. J. Ahrens, Sound velocities at high pressure and temperature and their geophysical implications, J. Geophys. Res., 97, 4503-4520,1992.

Duffy, T. S., and T. J. Ahrens, Temperature sensitivity of elastic wave velocity at high pressure: New results for molybdenum, Geophys. Res. Lett., 21, 473-476, 1994a.

Duffy, T. S. and T. J. Ahrens, Shock compression and release of polycrystalline magnesium oxide, High-Pressure
Science and Technology - 1993, edited by S. C. Schmidt, J. W. Shaner, G. A. Samara, and M. Ross, pp. 1107-1110, American Institute of Physics, New York, 1994b.

Grady, D. E., Processes occurring in shock wave compression of rocks and minerals, in High-Pressure Research: Applications to Geophysics, edited by M. H. Manghnani and S. Akimoto, pp. 389-438, Academic, San Diego, Calif., 1977.

Grady, D. E., Shock deformation of brittle solids, J. Geophys. Res., 85, 913-924, 1980.

Grady, D. E., and M. D. Furnish, Shock- and release-wave properties of MJ-2 grout, Rep. SAND88-1642, Sandia Natl. Lab., Albuquerque, N. M., 1988.

Graham, R. A., and W. P. Brooks, Shock-wave compression of sapphire from 15 to 420 kbar. The effects of large anisotropic compressions, J. Phys. Chem. Solids, 32, 2311-2330, 1971.

Heinz, D. L., and R. Jeanloz, The equation of state of the gold calibration standard, J. Appl. Phys., 55, 885-893, 1984.

Hemsing, W. F., Velocity sensing interferometer (VISAR) modification, Rev. Sci. Instrum., 50, 73-78, 1979.

Herrmann, W., Development of a high strain rate constitutive equation for 6061-T6 aluminum, Rep. SLA-730897, Sandia Natl. Lab., Albuquerque, N. M., 1974.

Isaak, D. G., O. L. Anderson, and T. Goto, Measured elastic moduli of single-crystal $\mathrm{MgO}$ up to $1800 \mathrm{~K}$, Phys. Chem. Mineral., 16, 704-713, 1989.

Isaak, D. G., R. E. Cohen, and M. E. Mehl, Calculated elastic and thermal properties of $\mathrm{MgO}$ at high pressures and temperatures, J. Geophys. Res., 95, 7055-7067, 1990.

Jackson, I., and H. Niesler, The elasticity of periclase to 3 GPa and some geophysical implications, in High Pressure Research in Geophysics, edited by S. Akimoto and M. H. Manghnani, pp. 93-133, Center for Academic Publishing, Tokyo, 1982.

Jeanloz, R., Shock wave equation of state and finite strain theory, J. Geophys. Res., 94, 5873-5886, 1989.

Jeanloz, R., Comment on "Pressure derivative of the bulk modulus" by A. M. Hofmeister, J. Geophys. Res., 97, $15,273,1992$.

Jeanloz, R., B. K. Godwal, and C. Meade, Static strength and equation of state of rhenium at ultra-high pressures, Nature, 349, 687-689, 1991.

Kipp, M. E., and D. E. Grady, Shock compression and release in high-strength ceramics, Rep. SAND89-1461, Sandia Natl. Lab., Albuquerque, N. M., 1989.

Kipp, M. E., and R. J. Lawrence, WONDY V - A onedimensional finite-difference wave propagation code, Rep. SA ND81-0930, Sandia Natl. Lab., Albuquerque, N. M., 1982.

Lawrence, R. J., and J. R. Asay, High-pressure multipleshock response of aluminum, in High Pressure Science and Technology, vol. 1, edited by K. D. Timmerhaus and M. S. Barber, pp. 88-98, Plenum, New York, 1979.

Lay, T., T. J. Ahrens, P. Olsen, J. Smyth, and D. Loper, Studies of the Earth's deep interior: goals and trends, Phys. Today, 43, 44-52, 1990.

Marsh, S. P., LASL Shock Hugoniot Data, 658 pp., University of California Press, Berkeley, 1980.

Mashimo, T., Shock compression studies on ceramic materials, in Shock Waves in Materials Science, edited by A. B. Sawaoka, pp. 113-144, Springer-Verlag, New York, 1993.

Mashimo, T., Y. Hanaoka, and K. Nagayama, Elastoplastic properties under shock compression of $\mathrm{Al}_{2} \mathrm{O}_{3}$ single crystal and polycrystal, J. Appl. Phys., 63, 327-336, 1988.

McQueen, R. G., S. P. Marsh, and J. N. Fritz, Hugoniot equation of state of twelve rocks, J. Geophys. Res., 72, 4999-5036, 1967.

McQueen, R. G., S. P. Marsh, J. W. Taylor, J. N. Fritz, and 
W. J. Carter, The equation of state of solids from shock wave studies, in High-Velocity Impact Phenomena, edited by R. Kinslow, pp. 294-419, Academic, San Diego, Calif., 1970.

Meade, C., and R. Jeanloz, Yield strength of $\mathrm{MgO}$ to 40 GPa, J. Geophys. Res., 9S, 3261-3269, 1988.

Mitchell, A. C., and W. J. Nellis, Shock compression of alnminum, copper, and tantalum, J. Appl. Phys., 52, 33633374, 1981.

Perez-Albuerne, E. A., and H. G. Drickamer, Effect of high pressures on the compressibilities of seven crystals having the $\mathrm{NaCl}$ or $\mathrm{CsCl}$ structure, J. Chem. Phys., 43, 13811387, 1965.

Romanowicz, B., Seismic tomography of the mantle, Annu. Rev. Earth Planet. Sci., 19, 77-100, 1991.

Rosenberg, Z., The response of ceramic materials to shock loading, in Shock Compression of Condensed Matter 1991, edited by S. C. Schmidt, R. D. Dick, J. W. Forbes, and D. G. Tasker, pp. 439-446, North-Holland, New York, 1992.

Sammis, C., D. Anderson, and T. Jordan, Application of isotropic finite strain theory to ultrasonic and seismological data, J. Geophys. Res., 75, 4478-4480, 1970.

Schreiber, E., and O. L. Anderson, Revised data on polycrystalline magnesium oxide, J. Geophys. Res., 73, 28372838, 1968.

Spetzler, H., and D. L. Anderson, Discrepancies in elastic constant data for $\mathrm{MgO}$ polycrystals and single crystals, J. Am. Ceram. Soc., 54, 520-525, 1971.

Sumino, Y., O. L. Anderson, and I. Suzuki, Temperature coefficients of elastic constants of single crystal $\mathrm{MgO}$ between 80 and $1300 \mathrm{~K}$, Phys. Chem. Mineral., 9, 38-47, 1983.

Svendsen, B., and T. J. Ahrens, Shock-induced temperatures of MgO, Geophys. J. Int., 91, 667-691, 1987.

Tagai, H., T. Zisner, T. Mori, and E. Yasuda, Preferred orientation in hot-pressed magnesia, J. Am. Ceram. Soc., 50, 550-551, 1967.

Weaver, J. S., T. Takahashi, and W. A. Bassett, Calculation of the P-V relation for sodium chloride up to 300 kilobars at $25^{\circ} \mathrm{C}$, in Accurate Characterization of the HighPressure Environment, edited by E. C. Boyd, NBS Spec. Publ. 326, 189-199, 1971.

Weidner, D. J., Y. Wang, and M. T. Vaughan, Yield strength at high pressure and temperature, Geophys. Res. Lett., 21, 753-756, 1994.

Zel'dovich, Ya. B., and Y. P. Raizer, Physics of Shock Waves and High-Temperature Hydrodynamic Phenomena, 916 pp., Academic, San Diego, Calif., 1967.

T. J. Ahrens, Seismological Laboratory, MS 252-21, California Institute of Technology, Pasadena, CA 91125.

(e-mail:tja@seismo.gps.caltech.edu)

T. S. Duffy, Geophysical Laboratory, Carnegie Institution of Washington, 5251 Broad Branch Road, N. W., Washington, DC 20015. (e-mail:duffy@gl.ciw.edu)

(Received April 6, 1994; revised July 28, 1994; accepted August 11, 1994.) 